\title{
A case study of ventilation shutdown with the addition of high temperature and humidity for depopulation of pigs
}

\author{
Angela Baysinger DVM, MS \\ Michael Senn DVM, MS \\ Jordan Gebhardt DVM, PhD \\ Christopher Rademacher DVM \\ Monique Pairis-Garcia DVM, PhD
}

From Veterinary and Consumer Affairs, Merck Animal Health, DeSoto, KS 66018 (Baysinger); High Plains Veterinary Consultants LLC, Newton, KS 67II4 (Senn); Department of Diagnostic Medicine/Pathobiology, College of Veterinary Medicine, Kansas State University, Manhattan, KS 66506 (Gebhardt); Department of Veterinary Diagnostic and Production Animal Medicine, College of Veterinary Medicine, lowa State University, Ames, IA 500II (Rademacher); and Department of Population Health and Pathobiology, College of Veterinary Medicine, North Carolina State University, Raleigh, NC 27606 (Pairis-Garcia).

Address correspondence to Dr. Baysinger (angela. baysinger@merck.com).

\begin{abstract}
CASE DESCRIPTION
In February 2020, a swine farm operating in multiple states throughout the Midwest began to evaluate emergency plans to respond to potential impacts of the COVID-19 pandemic.
\end{abstract}

\section{CLINICAL FINDINGS}

From February through April, extensive mitigation strategies were implemented in anticipation of market disruption. The farm consulted the AVMA Guidelines for the Depopulation of Animals to identify preferred methods for depopulation of swine; however, none of these methods were feasible. When the first US packing plant closed in April 2020 because of human COVID-19 infection, the farm began to evaluate whether ventilation shutdown plus (VSD+) could be used for depopulation.

\section{TREATMENT AND OUTCOME}

Through proof-of-concept trials, a method for ventilation shutdown with the addition of supplemental temperature and humidity was developed. A single location with 4 barns that could be retrofitted for the process was selected, and between April and June 2020, 243,016 pigs were depopulated (59,478 nursery and 183,538 finishing pigs). Mean \pm SD time to silent (the time when no sounds could be heard and no motion seen) was $55.4 \pm 14.5$ minutes for the nursery pigs and $65.0 \pm 18.1$ minutes for the finishing pigs. Only $728(0.300 \%)$ pigs required manual euthanasia at the end of the depopulation process.

\section{CLINICAL RELEVANCE}

Efficacy exceeded AVMA recommendations for the use of VSD+ (> 95\% mortality rate in < I hour). Findings could potentially guide the use of this method for mass depopulation in the event of a foreign animal disease outbreak or severe market disruption in the future. (J Am Vet Med Assoc 2021;259:415-424)
O n December 31, 2019, the Wuhan Municipal Health Commission in China publicly announced early signs of an outbreak of pneumonia in the city. By January 2020, cases had been reported from cities across China and in several other countries, and the cause had been identified as severe acute respiratory syndrome coronavirus 2 (SARS-CoV-2). The first known travel-related case entered the United States on January 15, and on January 31, Health and Human Services Secretary Alex Azar declared the outbreak a public health emergency. The first US death known to be a result of COVID-19 occurred in early February 2020 (Figure I). On March 11, the World Health Organization declared COVID-19 a global pandemic.

On February 10, 2020, the president of a swine farm operating in multiple states throughout the Mid-

\footnotetext{
ABBREVIATIONS

VSD Ventilation shutdown

VSD+ Ventilation shutdown with the addition of high temperature, a high concentration of carbon dioxide, or both

VSD $+\mathrm{TH}$ Ventilation shutdown with the addition of high temperature and humidity
}

west directed the farm's management team to evaluate emergency plans to respond to potential impacts of the COVID-19 pandemic on the care and management of the farm's pigs. Specific scenarios the management team was directed to evaluate included widespread illness among farm caretakers, feed mill drivers, maintenance crews, vaccination crews, and others; interruptions in supply chains for feed, medications, vaccines, and other vital goods; and slowdowns or closures of packing plants. Much of the preparatory work for this assessment had already been completed because the farm had previously established a crisis management team and participated in mock animal disease outbreak exercises with state and federal regulatory agencies because of concerns about the possible introduction of African swine fever. Importantly, during an infectious animal disease outbreak, US regulatory agencies and state and local authorities dictate animal movements and, if needed, depopulation procedures and provide personnel and resources. In this case, however, the farm was planning for potential infrastructure breakdowns occurring not as a result of an infectious animal disease outbreak but due to a public health disease outbreak, for which no governmental oversight or resources would be available. 


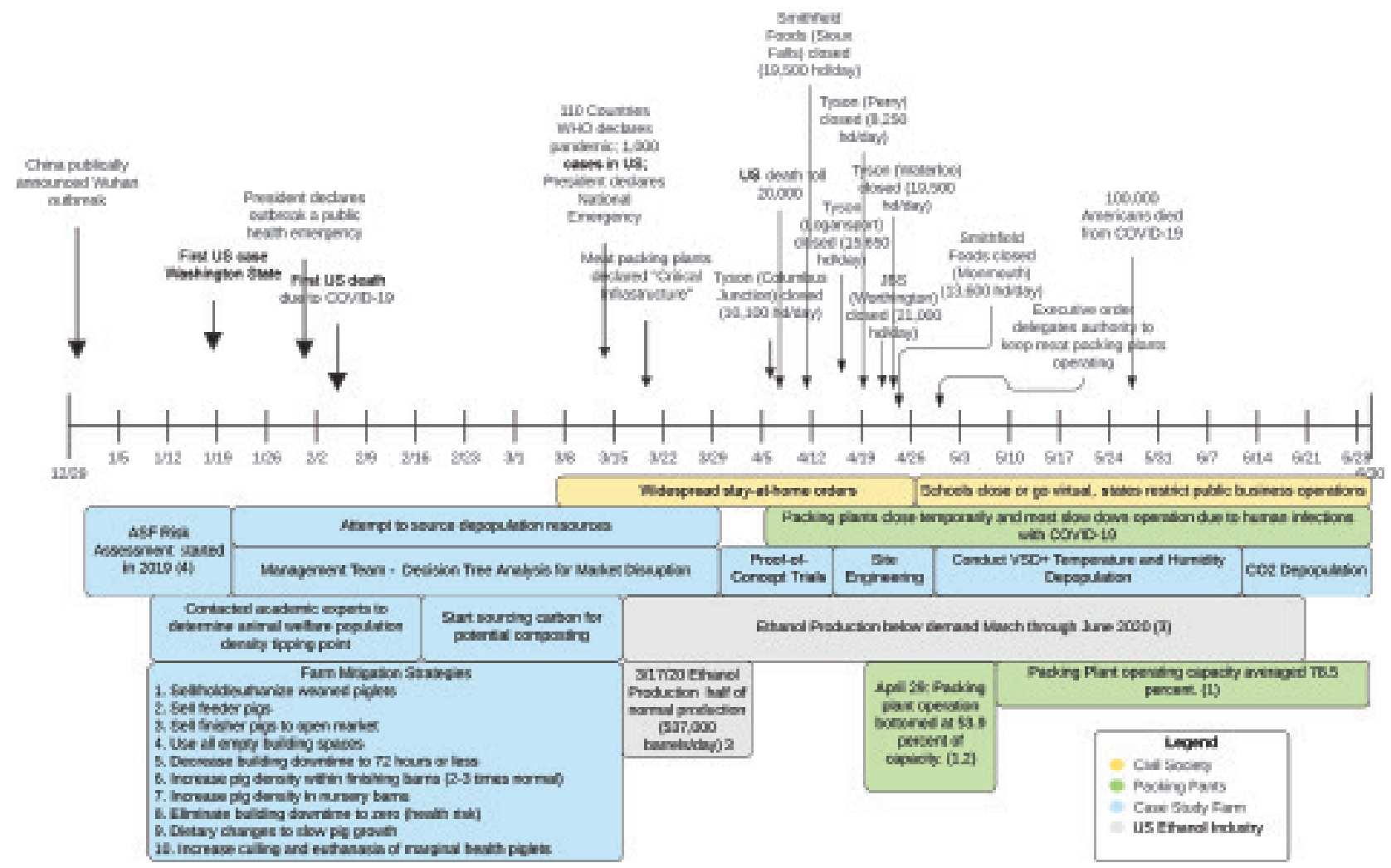

Figure I-Timeline depicting the impact of the COVID-19 pandemic on civil society, packing plants, the farm described in the present report (ie, case study farm), and the US ethanol industry (the source of $\mathrm{CO}_{2}$ for euthanasia) during January to July 2020.

In early March, farm management met with university-based academicians to discuss potential animal welfare impacts of overcrowding finishing pigs within barns in the event of packing plant closures or slowdowns. On the basis of previous research and published data, these experts counseled the farm on calculations that could be used to determine the tipping point when pig welfare would be jeopardized owing to inadequate food and water access and pig aggression.

In general, the floor space requirement for growing-finishing pigs can be estimated on the basis of body weight of the pigs, with heavier pigs requiring more floor space than lighter pigs. However, the relationship between body weight and floor space requirement is not linear ${ }^{5,6, a}$ and can be calculated on the basis of the following equation:

\section{Floor space requirement $\left(\mathrm{m}^{2}\right)=\mathrm{k} \times \mathrm{BW}^{0.66}$}

where $\mathrm{k}$ is a constant and BW is body weight expressed in kilograms. Under normal conditions, the farm described in the present report stocked growing-finishing pens to provide $0.632 \mathrm{~m}^{2} / \mathrm{pig}\left(6.8 \mathrm{feet}^{2} /\right.$ $\mathrm{pig}$ ), which equated to a $\mathrm{k}$ value of 0.047 . For the risk assessment, the farm decided to evaluate the number of pigs it could house with a $\mathrm{k}$ value of 0.025 , which would allow just enough space for all pigs to lie down, with some lying on their sternum and some lying partially on their side. From this calculation, the farm realized that if packing plants closed, it would need to begin depopulating within days after the closure.

Throughout February and March, farm management discussed and preemptively implemented numerous mitigation strategies to avoid animal welfare issues associated with overcrowding and decrease the risk that depopulation would be required. These mitigation strategies addressed all aspects of the pigs' management and included the following activities:

- Breeding of sows was decreased on farrowing farms. However, this had little impact on growing pig sites because the typical time from breeding to movement to growing pig sites is $\mathbf{5}$ months.

- Newborn and suckling piglets were sold or euthanized. Although this decreased the number of pigs that needed to be moved to growing pig sites, it did not decrease the number of pigs already at those sites.

- Feeder pigs were sold to other producers at light weights to remove pigs from growing pig sites. This had minimal impact because other producers were also concerned about possible packing plant closures.

- Finisher pigs were sold on the open market to other meat packers. This had minimal impact because many meat packers had minimal space for pigs from the open market and many meatpacking operations were closed 
or operating at reduced capacities because of COVID-19 infections among employees.

- All empty building spaces were used.

- Building downtime between groups of pigs was decreased to 72 hours or even 0 hours. Downtime represented the time typically used to clean, repair, and prepare the barns for new groups of pigs; therefore, decreasing downtime increased infectious disease risks for newly introduced pigs.

- Density was increased by 2 to 3 times in finishing barns while still providing sufficient space by stocking with young, lightweight pigs. This option was time limited as the pigs grew.

- Pig density was increased in nursery barns, while sufficient space was still provided. Again, this option was time limited as the pigs grew.

- Dietary changes were implemented to slow pig growth. This strategy was adopted before any of the options that required an increase in pig density. Nutritionists were consulted to develop diets that would decrease growth but provide satiation to avoid the stress associated with hunger.

- Culling and euthanasia of marginally healthy pigs were increased. This provided only a minimal decrease in overall pig numbers.

- $\quad$ Pigs were sold to private individuals and donated to charities. This option was extensively exploited but did not substantially reduce growing pig numbers.

- Options for housing in nontraditional locations were explored. The farm attempted to source available poultry locations, but none were available. Housing pigs outdoors was not considered viable because of inadequate containment (fencing); impracticalities associated with providing feed, water, and shelter; liability concerns; and potential environmental impacts. Additionally, low nighttime temperatures would have threatened animal welfare.

When President Trump declared the pandemic a national emergency on March 13, 2020, the farm initiated a search for resources needed to conduct mass depopulation. The AVMA Guidelines for the Depopulation of Animals ${ }^{7}$ were consulted to identify methods classified as preferred or permitted in constrained circumstances for depopulation of swine. In addition, various market sources and state and federal agencies were contacted to inquire about the availability of equipment needed to implement each of the preferred methods, such as captive bolt guns and charges, guns and ammunition, carbon dioxide, and electrocution equipment.

The farm evaluated the feasibility of each depopulation method, and methods were ranked on the basis of animal welfare impact, aesthetics of the process, operator safety, timeliness (ie, number of animals that could be depopulated in a set amount of time), and environmental impact of the carcasses
(Figure 2). Given the large number of pigs the farm projected would need to be depopulated, human safety and training considerations, and aesthetics for caretakers and the public, carbon dioxide was selected as the farm's first method of choice. ${ }^{7,8}$

Carbon dioxide is a byproduct of the ethanol industry in the Midwest; therefore, the farm contacted all ethanol facilities in the Midwest to determine the quantity of carbon dioxide available to them for implementing mass depopulation. However, many ethanol plants were not operating because stay-at-home orders had decreased demand for ethanol-containing fuels, ${ }^{3}$ and ethanol facilities that were operating could not supply the quantity of carbon dioxide needed because of regular customer demand.

For these reasons, the farm began to research the feasibility of other preferred methods of depopulation. Both captive bolt and gunshot were considered unfeasible because of a lack of availability of the necessary resources (ie, captive bolt guns and charges and guns and ammunition). Electrocution was not considered a viable option because a farm-tested system had not been built in the United States, and engineers who were consulted on the topic suggested that it would take $\geq 6$ months to develop a prototype. Finally, administering an anesthetic overdose was considered unacceptable because, given the large number of pigs that potentially needed to be depopulated, injecting each individual pig would not be practical; only veterinarians can administer anesthetic drugs; and carcass disposal would be impractical because carcasses could not be rendered for use in animal feed, owing to residual drug in the carcasses, and could not be composted, owing to the risk of wildlife consumption of residual drug and environmental contamination.

On April 6, 2020, the first meatpacking plant in the United States closed because of COVID-19 among its workers. That same day, the farm management and veterinarians determined that they needed to evaluate whether some form of VSD could be used for depopulation. Although instances of ventilation failure had been documented, ${ }^{9,10}$ no established or documented protocols for VSD+ existed. Therefore, farm veterinarians contacted several university-based swine veterinarians and engineers to develop a small proof-of-concept study to determine the feasibility and effectiveness of the VSD+ method.

On April 15, 2020, an initial proof-of-concept trial was performed with a livestock trailer that had been fitted with heaters and temperature-monitoring devices. The veterinarian reported that the method was 90\% effective within an hour with heat alone but did not meet the AVMA depopulation guidelines recommendation that VSD be used only if it resulted in a $>$ $95 \%$ death rate in $<1$ hour.

The veterinarians and engineers reviewed human and animal research on hyperthermia ${ }^{11,12}$ and determined that humidity would theoretically expedite the hyperthermic process. On April 22, 2020, a 


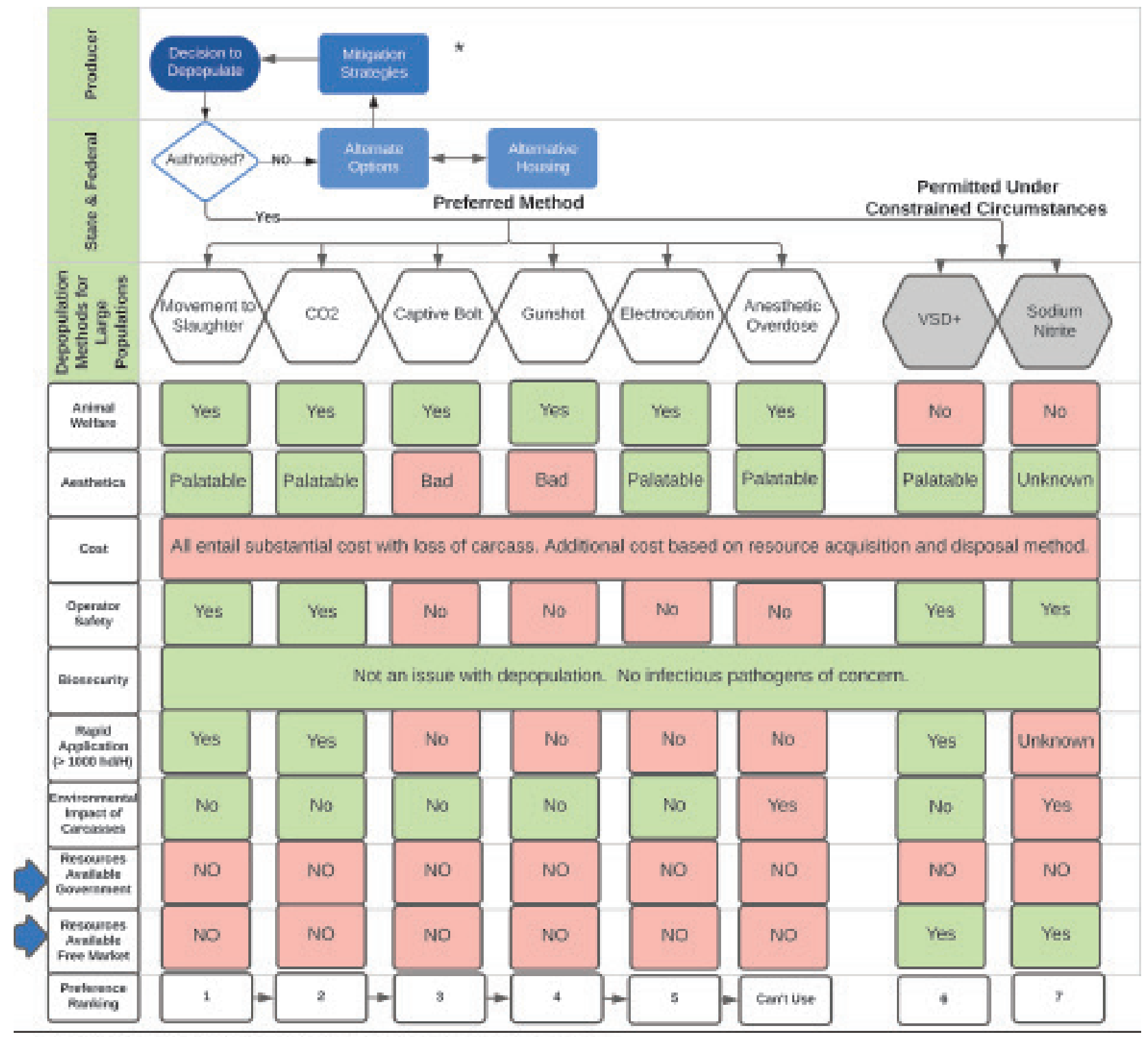

Fed indicates the case farm calegoriaed this option with a negathe response.

Grean incieales the case farm cabegerized this optien with a pesitive respense.

"Mitgation strategies are found in Figure 2.

Figure 2-Assessment performed by the case study farm between February and April 2020 of the animal welfare impacts, aesthetics, operator safety, rapid application for large numbers of animals, environmental impact, and availability of resources from the government and free market for various methods of depopulation of swine classified in the AVMA Guidelines for the Depopulation of Animals ${ }^{7}$ as preferred or permitted in constrained circumstances and the ultimate farm ranking of each depopulation method. Blue arrows indicate important limiting factors affecting the farm's choice of depopulation method.

second proof-of-concept trial was conducted with the same livestock trailer fitted with heaters to increase the internal temperature but with the addition of a low-pressure steam generator to supply supplemental humidity. In this trial, the mortality rate was $100 \%$ within 60 minutes.

While the proof-of-concept trials were going on, farm management evaluated the logistics of applying the VSD+TH method. The farm had growing pig barns located throughout the Midwest, and engineering all of these barns across multiple states to ensure a consistent, efficacious process was not deemed possible. Ad- ditionally, the farm wanted to minimize the emotional impact that depopulation of healthy pigs could have on caretakers across all of the growing pig locations. A further consideration was management of the carcasses to meet state environmental regulations if composting was used. The farm management identified a single 4-barn site that could be engineered for VSD+TH, with all pigs to be transported to this location.

On April 17, 2020, the farm's resource acquisition manager started sourcing the fibrous material and machinery needed to compost a calculated 200,000 pigs (projecting a possible packing plant closure or slow- 
down of approximately 20 days). In addition, on April 22, 2020, after the second proof-of-concept trial was successful, all materials needed to seal the 4 barns, remove existing equipment from the barns, and retrofit the barns for carcass removal were sourced, and steam generation equipment was procured. Retrofitting of the barns was completed by April 28, 2020.

The site for the depopulation process consisted of 4 barns built to operate as tunnel-ventilated, singleroom structures with shallow manure pits. As part of the retrofitting of each barn, all penning, gating, feeders, and waterers were removed. The shallow manure pits were filled with gravel and dirt beneath the slatted floor to eliminate air space in the manure pit and allow skid loaders to move within the barn for carcass removal without breaking through the slatted floor. Two large doorways were cut into each barn's exhaust end to accommodate the skid loaders. All ceiling inlets and wall seams and the curtain air inlet on the sidewall were sealed.

The original heaters ${ }^{\mathrm{b}}$ were moved from inside the building to an external location so that they could operate efficiently and avoid the risk of malfunction associated with humidity inside the barn. Commercial-grade mobile steam generators ${ }^{\mathrm{c}}$ typically used in the railroad industry to heat railcars were obtained. Two steam generators were plumbed for each barn, and commercial steam tubing was connected to the barn to enter at the ceiling level in 3 locations. This design aimed to ensure that steam would enter the room at ceiling level, move across the ceiling, and filter down to the pig level, avoiding direct contact with the pigs (Figure 3 ).

On April 29, 2020, a sufficient number of meatpacking plants were closed or operating at reduced capacity because of human illness or absenteeism that the US swine slaughter system was operating at only
$53.9 \%$ of its typical capacity. ${ }^{2}$ On April 30, 2020, the farm conducted its first VSD+TH operation in a single barn at the depopulation site. The efficacy of the initial 1-barn cycle was $99.4 \%$, with 1,461 of 1,470 pigs killed during the farm's depopulation process. Nine (0.61\%) pigs were euthanized by means of captive bolt at the end of the first whole-barn VSD+TH process.

Subsequently, between April and June 2020, a total of 243,016 pigs were depopulated, including 59,478 nursery pigs and 183,538 finishing pigs. Two veterinarians were on location each time depopulation was performed. Each veterinarian oversaw 2 barns and was responsible for coordinating the VSD $+\mathrm{TH}$ process with a team of 12 caretakers ( 6 caretakers/barn). The veterinarian managed the process from a control room and was responsible for controlling the heaters, monitoring the barn's electronic temperature sensors, documenting all events during the process, and monitoring 2 wireless humidity and temperature sensors and recorders. ${ }^{\mathrm{d}}$ The veterinarian modified the heat control settings on the basis of readings from the barn's electronic sensors and used 2 -way radios to communicate with the steam generator operators and team members.

On depopulation days, the site team would conduct a briefing with the veterinarians in charge before the depopulation process began. Team members reviewed the schedule for the day, identified any operational risks, reviewed the protocol, and appointed barn teams and responsibilities. The baseline protocol was posted in the control rooms where the veterinarians were located for reference and was adjusted as needed (Appendix). Veterinarians in charge of the process were both farm employees and veterinarians in private practice. They were responsible for monitoring animal welfare during pig transportation and movement, controlling the variability of the pro-

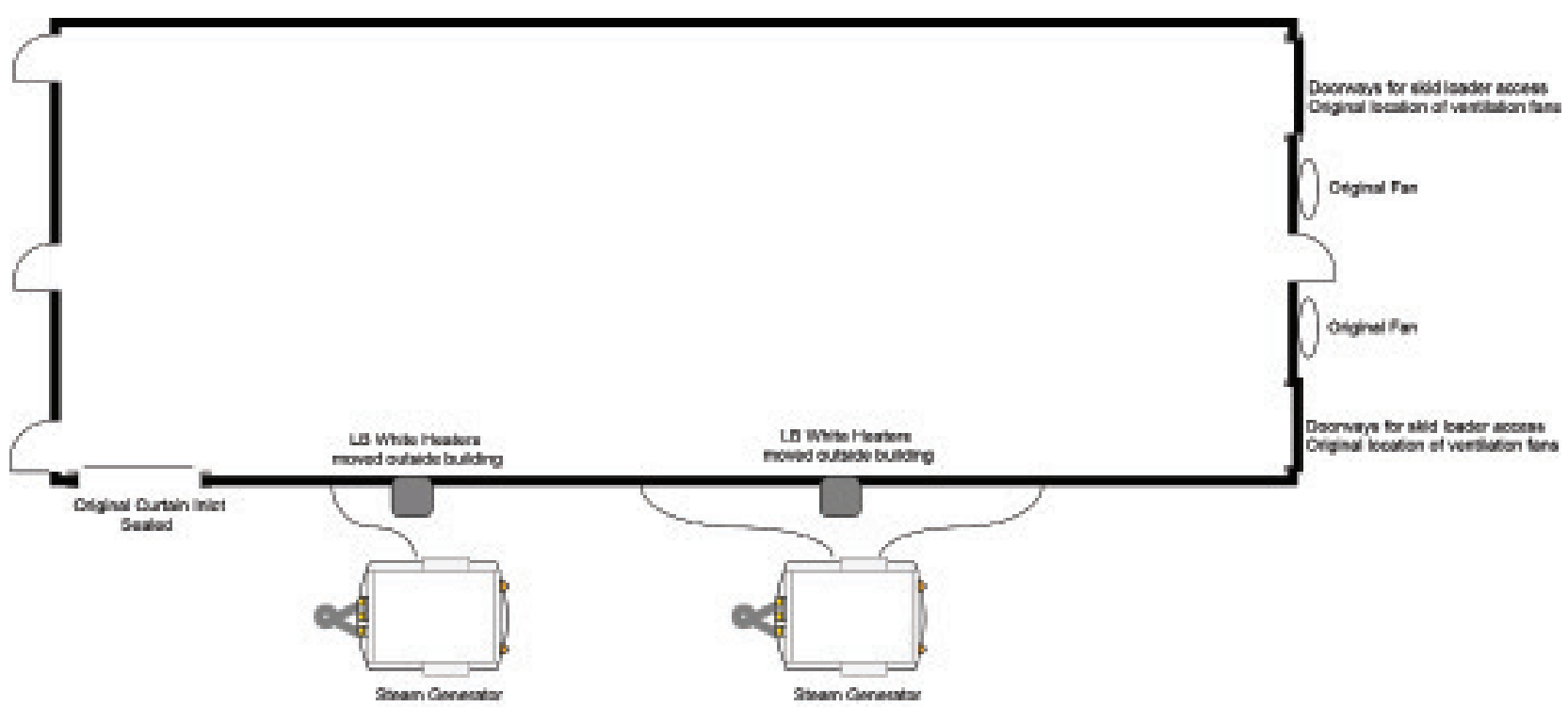

Figure 3-Diagram of a barn used for depopulation of swine following retrofitting as a single open room with entry doors for skid loaders to allow carcass removal, heaters relocated outside the barn, and the addition of 2 steam generators. 
cess, documenting all procedures, and verifying that each animal was dead. ${ }^{13-16}$

For the present report, written reports from all veterinarians involved in the depopulation process and electronic data logger information were obtained and analyzed. For purposes of analysis, a cycle was defined as a single group of pigs in a single barn that underwent the depopulation process. Data collected for each cycle by the veterinarian in charge consisted of the following information:

- Total cycle time (ie, time from door closure to the time doors were reopened).

- Total number of pigs.

- Time to time 0 . Time 0 was defined as the time when the mean barn temperature reached $54^{\circ} \mathrm{C}$ $\left(130^{\circ} \mathrm{F}\right)$. This threshold temperature was determined during the proof-of-concept trials and was the temperature at which the animals began to show signs of increased respiration followed by open-mouth breathing. Steam generation was begun at time 0 , and humidity was maintained at $\geq$ $90 \%$ until no sounds were heard within the barn.

- Time to silent. Time to silent was defined as the time from time 0 to the time when no sounds could be heard within the barn and no motion was seen. The veterinarian overseeing the operation was located in a control room between the 2 barns for which they were responsible and would walk a short distance in the connecting hallway to physically evaluate each barn during the cycle. The veterinarian would open the people entry door, look into the barn, observe the animals for activity, and listen for sounds of breathing or panting. Additionally, the barn team positioned outside the building would look through the windows to observe the pigs for any motion and listen for any sounds.

- Number of animals that had to be euthanized at the end of each cycle. At the end of each cycle, the veterinarian, a barn owner, and 1 additional person walked through the barn and examined each animal for signs of consciousness. Any animal with signs of consciousness was immediately euthanized with a captive bolt device. Individuals who removed carcasses from the barn were also responsible for monitoring for any signs of consciousness.

Data were entered into a spreadsheet database, ${ }^{\mathrm{e}}$ and descriptive statistics and frequency tables were generated with standard software. ${ }^{\text {f }}$ Additionally, hourly ambient weather conditions were gathered from historical databases for the nearest weather recording station, a and ambient weather data for the nearest hour to cycle initiation were extracted. Data visualization was performed with data visualization software $^{\mathrm{h}}$ and an associated software programming language. ${ }^{i}$ Density histograms were generated with a commercially available software packagej by means of a kernel-smoothed density estimate.

A total of 16 cycles were used for depopulation of the 59,478 nursery pigs. Time to silent was documented for 14 of these 16 cycles, and mean time to silent was 55.4 minutes (SD, 14.5 minutes; range, 40.0 to 80.0 minutes; Table I; Figure 4). A total of 122

Table I-Descriptive statistics for the outcome of VSD+TH for depopulation of pigs at a farm in the Midwest during the COVID- 19 pandemic.

\begin{tabular}{|c|c|c|c|c|c|c|}
\hline Variable & No. of cycles* & Minimum & Mean & Median & Maximum & SD \\
\hline \multicolumn{7}{|l|}{ Nursery pigs } \\
\hline Efficacy $(\%)$ & 16 & 99.9 & 100.0 & 100.0 & 100.0 & 0.02 \\
\hline No. of pigs/cycle & 16 & $3,376.0$ & $3,7 \mid 7.4$ & $3,611.0$ & $4,111.0$ & 256.7 \\
\hline Total time (min) & 16 & 70.0 & 90.4 & 91.0 & 110.0 & 11.3 \\
\hline Time to time 0 ( $(\mathrm{min})$ & 16 & 15.0 & 24.0 & 24.0 & 34.0 & 5.6 \\
\hline Time to silent (min) & 14 & 40.0 & 55.4 & 49.0 & 80.0 & 14.5 \\
\hline Maximum cycle temperature $\left({ }^{\circ} \mathrm{C}\right)$ & 15 & 63.9 & 69.2 & 70.1 & 73.3 & 3.2 \\
\hline Ambient humidity (\%) & 16 & 64.3 & 73.7 & 73.4 & 87.8 & 7.7 \\
\hline Wind speed $(\mathrm{m} / \mathrm{s})$ & 16 & 5.8 & 9.6 & 9.8 & 13.3 & 3.4 \\
\hline \multicolumn{7}{|l|}{ Finishing pigs } \\
\hline Efficacy $(\%)$ & 122 & 90.5 & 99.6 & 99.9 & 100.0 & 1.01 \\
\hline No. of pigs/cycle & 122 & $\mathrm{I}, 100.0$ & $\mathrm{I}, 504.4$ & $\mathrm{I}, 521.0$ & $\mathrm{I}, 754.0$ & 144.9 \\
\hline Total time (min) & 122 & 66.0 & II0.3 & I 10.0 & $15 \mid .0$ & 15.6 \\
\hline Time to time $0(\mathrm{~min})$ & 120 & 15.0 & 30.6 & 28.0 & 94.0 & 11.3 \\
\hline Time to silent (min) & 120 & 14.0 & 65.0 & 63.0 & 106.0 & I8.| \\
\hline Ambient temperature $\left({ }^{\circ} \mathrm{C}\right)$ & 122 & 0.5 & 12.2 & 13.0 & 25.5 & 5.9 \\
\hline Ambient humidity (\%) & 122 & 19.7 & 63.4 & 64.1 & 96.8 & 22.2 \\
\hline Wind speed $(\mathrm{m} / \mathrm{s})$ & 122 & 0.0 & 9.1 & 8.6 & 17.2 & 4.3 \\
\hline
\end{tabular}

*Number of cycles for which data were available.

A total of 243,016 pigs were depopulated by the farm, consisting of 59,478 nursery pigs ( 16 depopulation cycles, with each cycle defined as a single group of pigs in a single barn) and 183,538 finishing pigs (I22 cycles). Efficacy was defined as the percentage of pigs in each cycle that were dead by the end of a cycle. Total time was the total time for each cycle from when the barn was closed and the heaters were turned on to when the heaters and steam generators were turned off and the barn doors were opened. Time to time 0 was the time from when the barn was closed and the heaters were turned on to when the mean barn temperature reached $54^{\circ} \mathrm{C}$ and the steam generators were turned on. Time to silent was the time from time 0 to when no sounds could be heard within the barn and no motion was seen. 
cycles were used for depopulation of the 183,538 finishing pigs. Time to silent was documented for 120 of these cycles, and mean time to silent was 65.0 minutes (SD, 18.1 minutes; range, 14.0 to 106.0 minutes).

Of the 16 nursery cycles, only 4 were $<100 \%$ efficacious, and only 6 of the $59,478(0.010 \%)$ nursery pigs were manually euthanized. All but 1 of the 122 finishing pig cycles were $>95 \%$ efficacious, and only 722 of the 183,538 (0.393\%) finishing pigs were manually euthanized (Figure 5). In total, only 728 of the $243,016(0.300 \%)$ pigs were manually euthanized.

The state regulatory authority was consulted regarding methods of carcass disposal. Because infectious disease was not a concern, the farm was granted permission by the state regulatory agency to compost or render all carcasses. The farm sent 168,016 (69\%) of the carcasses to multiple rendering companies throughout the Midwest. The remaining 75,000 carcasses were composted at the depopulation site under the oversight of the state regulatory agency. The composted materials were expected to be used locally for agricultural crop production when the composting process was complete.

Most importantly, the cost of depopulation was not a consideration in determining the method used. Direct costs associated with the VSD+TH method were $\$ 10,490,480$, which consisted of the costs of retrofitting and engineering the 4 barns $(\$ 12,500 / \mathrm{barn})$, resources and supplies for the depopulation process $(\$ 900,000)$, rendering of 168,016 carcasses $(\$ 30 / \mathrm{car}-$ cass), and composting of 75,000 carcasses (\$60/carcass). Additional costs represented, but not included in the reported costs, were the loss of the potential market value of the 243,016 pigs; the costs of labor, facilities, supplies, equipment, and transportation; the cost of the $4 \mathrm{barns}$; and the cost of the mitigation strategies employed prior to depopulation. An unaccountable cost was the emotional impact on the people involved.

Because depopulation was not conducted for a reportable animal disease, no governmental financial support was available at the time of depopulation to offset the costs of the animals' economic value, depopulation process, labor, loss of the 4 barns, or carcass disposal.

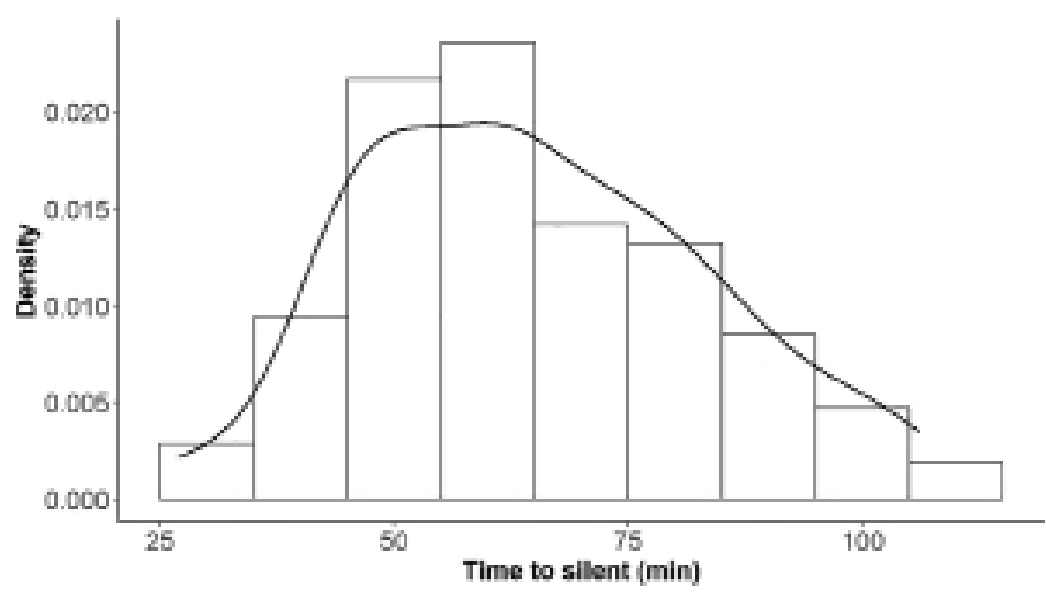

Figure 4-Distribution (bars) and probability density function (solid line) of time to silent (defined as time when mean barn temperature reached $54^{\circ} \mathrm{C}$ to time when no sounds could be heard and no motion seen) for the 134 depopulation cycles (with each cycle defined as a single group of pigs in a single barn) of nursery ( $n=14$ cycles) and finishing (I20 cycles) pigs. Each point along the probability density function represents the probability that a cycle of pigs would have the corresponding time to silent; area under the probability density function curve represents a probability of $\mathrm{I}$.
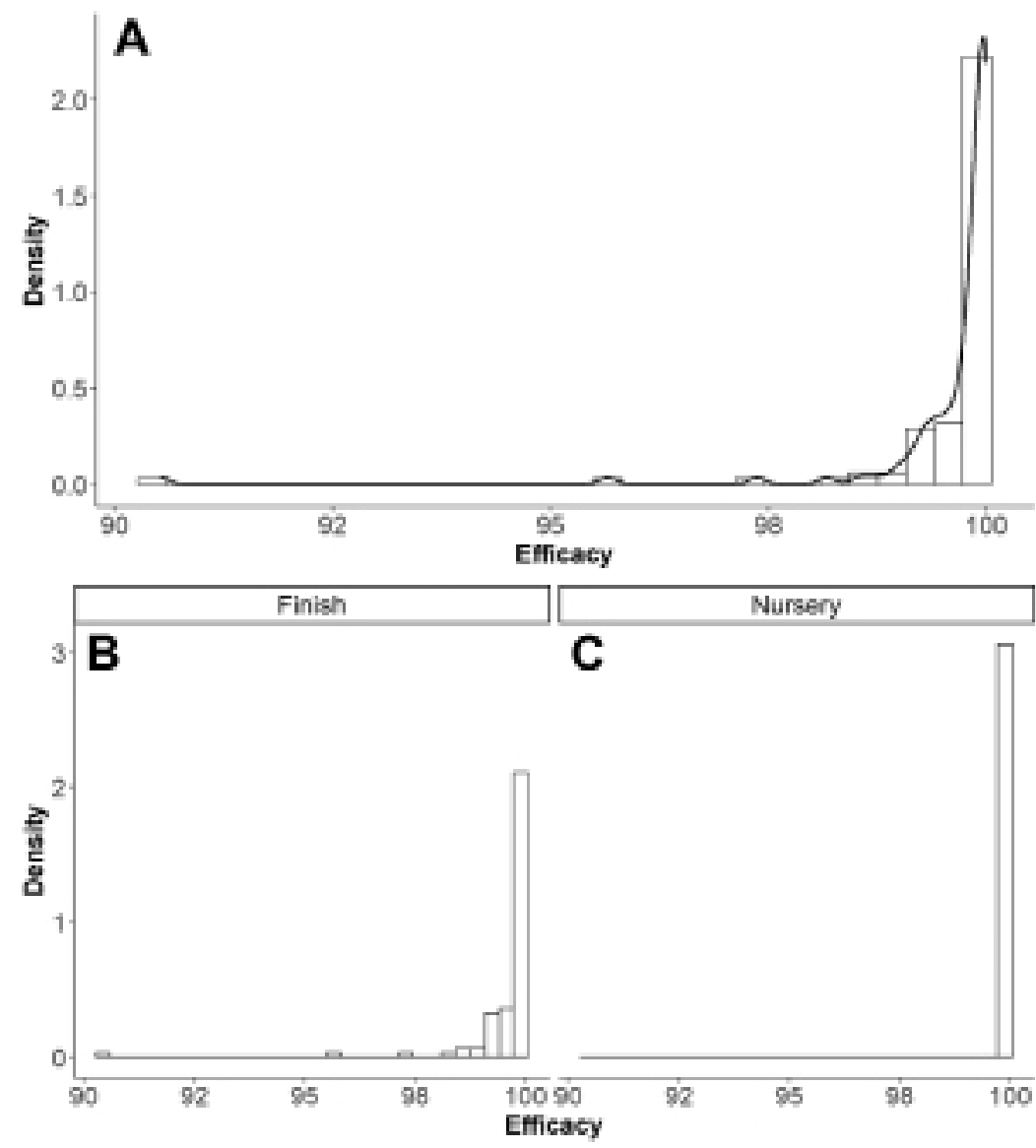

Figure 5-Density plot of cycle mortality efficacy (percentage of pigs in each cycle that were dead by the end of a cycle) for 243,016 pigs (I38 cycles) depopulated by the case study farm (A), consisting of 183,538 finishing pigs (I22 cycles; $B$ ) and 59,478 nursery pigs ( 16 cycles; $C$ ). The vertical axis represents the probability density or probability per unit on the horizontal axis. 


\section{Discussion}

In 2020, the closure of processing plants and associated animal movement restrictions due to the COVID-19 pandemic led to a requirement for mass depopulation of an estimated 1 million pigs in the United States. Mass depopulation encompasses the emergency killing of large numbers of animals and is implemented only in emergency situations and only with governmental oversight. Mass depopulation should never be implemented under normal circumstances, and methods used must balance the critical need for a rapid response with requirements for human safety and animal welfare. In the United States, studies have not yet validated depopulation methods that could be used on-farm for swine at a large scale. ${ }^{17}$ The information provided in the present report was derived from the depopulation of $>240,000$ pigs between April and June 2020 necessitated by the COVID-19-related closure of packing plants. The lack of published work and limited guidelines on effective depopulation methods in the United States encouraged our team to document this novel case, including the decision-making process and the protocol and outcomes of the VSD+TH method.

Up until 2020, US swine producers have not needed to implement depopulation on a large scale and have been able to manage disease outbreaks (eg, porcine epidemic diarrhea) with on-farm euthanasia techniques approved by the AVMA. ${ }^{18}$ For the farm described in the present report, however, these techniques were not considered an option because of the large number of pigs that would need to be euthanized over such a short period of time.

The AVMA depopulation guidelines ${ }^{7}$ list various preferred methods for depopulation of pigs and several methods that it classifies as "permitted in constrained circumstances." One of those methods classified as permitted in constrained circumstances is VSD+. Notably, the VSD+TH method used by the farm in the present report was an enhancement of the $\mathrm{VSD}+$ method, in that not only high temperatures but also high humidity were used after heat alone was found to be insufficient in a proof-of-concept trial to meet the AVMA recommendation that VSD+ be used only if it resulted in a $>95 \%$ death rate in $<1$ hour.

To summarize, COVID-19-related closure of processing facilities caused movement restrictions of healthy pigs because there were no alternative locations for slaughter and no alternative locations to house the pigs until processing facilities resumed operation. These movement restrictions compromised the welfare of pigs housed in overstocked barns and resulted in a domino effect impacting pigs and producers throughout the entire production chain. ${ }^{19-26}$ The combination of COVID-19 among processing facility workers and restricted space on swine farms forced many producers to make the decision to depopulate.

Importantly, once packing plant closures started, there was no way to predict how long the plants would remain closed or when they would be able to return to full capacity after reopening. On April 29, 2020, the US swine slaughter system was operating at $53.9 \%$ of its typical capacity, ${ }^{2}$ and guidance from the CDC and Occupational Safety and Health Administration suggested that capacity could remain below prepandemic levels for the balance of 2020 and into 2021. ${ }^{27}$

Finally, as the farm described in the present report was preemptively preparing for possible depopulation, farm managers attempted to allocate resources needed for methods classified as preferred in the AVMA depopulation guidelines. ${ }^{7}$ However, the farm soon realized that the pandemic had affected all resource markets and that there was limited availability of ammunition and captive bolt equipment, no commercially available equipment for on-farm electrocution, and an insufficient amount of carbon dioxide for the number of pigs the farm would need to depopulate. Without any other options available, the farm turned to VSD+TH.

In total, only 728 of the $243,016(0.300 \%)$ pigs were manually euthanized following VSD+TH. Data collected during the depopulation process indicated that the process-controlled VSD+TH procedure was extremely effective under the conditions on the farm and exceeded the expectations for mass depopulation of swine described in the AVMA depopulation guidelines, ${ }^{7}$ which state that VSD should only be used if it results in a $>95 \%$ death rate in $<1$ hour. For this farm, the VSD+TH procedure involved closing the barn, shutting all inlets, turning off all fans, and providing supplemental heat and humidity. The addition of high humidity was expected to accelerate the hyperthermia process through the generation of latent heat, resulting in hyperthermia and subsequent heatstroke leading to death. Importantly, the farm incorporated an in-depth decision tree, extensive engineering, extreme process control, veterinary oversight, and multiple data collection methods to monitor and evaluate the VSD+TH procedure. The engineering modifications to the barns needed to conduct VSD+TH would not permit use for pig production in the future, and the barns were scheduled for demolition.

The farm described in the present report elected to perform all depopulation procedures at a single location, transporting 243,016 pigs from their growing farm of origin to the designated depopulation site. The following benefits were associated with performing depopulation at a single location:

- Only a limited number of barns were needed to be engineered and retrofitted for VSD+TH. Because barns could never be used for growing pigs again, only a limited number of barns at a single location were destroyed.

- A designated team of caretakers could be assigned to the depopulation location. This minimized human exposure to the depopulation process. Professional counseling could be offered to the designated team instead of caretakers at all growing pig farms of origin.

- Regulatory permission could be obtained to conduct carcass composting at the same site where depopulation occurred. This minimized addition- 
al transportation of carcasses and the resources (eg, fiber material and machinery) needed for the composting process.

Of course, the use of a single location would likely not be possible in the event depopulation would be required to respond to an infectious animal disease outbreak because transportation of live animals or carcasses would be a pathogen transmission risk. However, consideration could be given to the use of a centralized depopulation location for healthy animals in a stop movement zone if the welfare of those animals was at risk because of overcrowding or a lack of access to feed or water.

Results for the farm described in the present report indicated that the VSD+TH method exceeded the requirements outlined in the AVMA depopulation guidelines of a $>95 \%$ mortality rate in $<1$ hour. However, to successfully use VSD+TH for mass depopulation of swine, extensive planning must be performed, a process-controlled protocol must be in place, veterinary oversight of the operation must be established, animal welfare must be ensured, and the facility must be extensively engineered. This depopulation method is a last resort, and VSD+TH was only used because other preferred methods were not possible during this emergency. However, VSD+TH is not a method conducted without personal and societal impact.

Throughout the entire process, the farm described in the present report took extensive steps to evaluate the depopulation method and ensure that it was conducted with all consideration for the animals, people, environment, and social perspectives, all with limited instructions and resources. The farm worked with state veterinarian and regulatory agency officials to obtain guidance and ensure transparency throughout the depopulation process. Additionally, animal scientists, engineers, and veterinarians from multiple universities worked collaboratively with the farm throughout the process. The farm openly allowed veterinarians from practices throughout the swine industry, veterinarians from nongovernmental organizations, and animal health and welfare experts to observe and provide input into the depopulation process.

\section{Acknowledgments}

No third-party funding or support was received in connection with this study or the writing or publication of the manuscript. The authors declare that there were no conflicts of interest.

\section{Footnotes}

a. Johnson L, Li Y, Minnesota Swine Extension, University of Minnesota, Saint Paul, MN: Personal communication, 2021.

b. Direct-fired LB White Guardian, LB White Co, Onalaska, Wis c. Steam-Flo 20, Sioux Corporation, Beresford, SD.

d. LogTag, MicroDAQ.com Ltd, Contoocook, NH.

e. Excel, Microsoft, Redmond, Wash.

f. SAS, version 9.4, SAS Institute Inc, Cary, NC.

g. Visual Crossing. Available at: www.visualcrossing.com. Accessed December 2020

h. RStudio environment, version 1.3.1093, RStudio Team, Boston, Mass

i. R programming language, version 4.0.2, R Core Team, Vienna, Austria.

j. $\quad$ ggplot2 package, version 3.3.2, RStudio Team, Boston, Mass.

\section{References}

1. USDA. Quarterly hogs and pigs report, March 2021. Available at: https://usda.library.cornell.edu/concern/publications/ rj430453j?locale=en. Accessed Mar 6, 2021

2. Kansas State University. Weekly hog slaughter. Available at: www.agmanager.info/livestock-meat/livestock-marketingcharts/weekly-hog-slaughter. Accessed Nov 6, 2020.

3. US Energy Information Administration. Weekly Midwest (PADD 2) oxygenate plant production of fuel ethanol (thousand barrels per day). Available at: www.eia.gov/dnav/pet/ hist/LeafHandler.ash $\mathrm{x} ? \mathrm{n}=\mathrm{PET} \& \mathrm{~s}=\mathrm{W} \_$EPOOXE_YOP_R20_ MBBLD\&f=W. Accessed Jan 29, 2021

4. Case study farm documentation and notes, 2020 .

5. Gonyou HW, Brumm MC, Bush E, et al. Application of broken-line analysis to assess floor space requirements of nursery and grower-finisher pigs expressed on an allometric basis. J Anim Sci 2006;84:229-235.

6. Petherick JC, Phillips CJC. Space allowances for confined livestock and their determination from allometric principles. Appl Anim Behav Sci 2009;117:1-12.

7. Leary S, Anthony R, Gwaltney-Brant S, et al. AVMA guidelines for the depopulation of animals: 2019 edition. Schaumburg, Ill: AVMA, 2019;43-45.

8. American Association of Swine Veterinarians. Recommendations for the depopulation of swine. Available at: www.aasv. org/Resources/welfare/depopulation_recommendations. pdf. Accessed Jun 15, 2020.

9. Bird N. Ventilation failure alarms: 2 case studies. Available at: www.dicam.co.uk/wp-content/uploads/filebase/research/ Case_Study_2_ventilation_failure_incidents.pdf. Accessed Oct $4,2021$.

10. Robert MJ, Shaffer CS, Funk TL, et al. Carbon dioxide and temperature change due to ventilation failure, in Proceedings. Swine Housings II Conference, 2003;9-15.

11. Bouchama A, Knochel J. Heat stroke. $N$ Engl J Med 2002;346:1978-1988

12. Robertshaw D. Mechanisms for the control of respiratory evaporative heat loss in panting animals. I Appl Pbysiol (1985) 2006;101:664-668

13. EFSA Panel on Animal Health and Welfare. Welfare of pigs during killing for purposes other than slaughter. EFSA $J$ 2020;18:e6195.

14. World Organisation for Animal Health (OIE). Terrestrial animal bealth code. 28th ed. Paris: World Organisation for Animal Health, 2019

15. Gavinelli A, Kennedy T, Simonin D. The application of humane slaughterhouse practices to large-scale culling. Rev Sci Tech 2014;33:291-301.

16. Berg $\mathrm{C}$. The need for monitoring farm animal welfare during mass killing for disease eradication purposes. Anim Welf 2012;21:357-361.

17. Arruda AG, Beyene TJ, Kieffer J, et al. A systematic literature review on depopulation methods for swine. Animals (Basel) $2020 ; 10: 2161$.

18. Leary S, Underwood W, Anthony R, et al. AVMA guidelines for the euthanasia of animals: 2020 edition. Schaumburg, Ill: AVMA, 2020.

19. Bargen LL, Whiting TL. Time to critical overcrowding of Manitoba swine barns in the event of restriction on animal movement. Can Vet J 2002;43:855-862.

20. Crispin SM, Roger PA, O'Hare H, et al. The 2001 foot and mouth disease epidemic in the United Kingdom: animal welfare perspectives. Rev Sci Tech 2002;21:877-883.

21. East IJ, Roche SE, Wicks RM, et al. Options for managing animal welfare on intensive pig farms confined by movement restrictions during an outbreak of foot and mouth disease. Prev Vet Med 2014;117:533-541.

22. Howden KJ, Brockhoff EJ, Caya FD, et al. An investigation into human pandemic influenza virus (H1N1) 2009 on an A1berta swine farm. Can Vet J 2009;50:1153-1161.

23. Laurence CJ. Animal welfare consequences in England and Wales of the 2001 epidemic of foot and mouth disease. Rev Sci Tech 2002;21:863-868. 
24. Mangen MJ, Burrell AM, Mourits MCM. Epidemiological and economic modelling of classical swine fever: application to the $1997 / 1998$ Dutch epidemic. Agric Syst 2004;81:37-54

25. Weng HY, Yadav S, Olynk Widmar NJ, et al. Modelling the time at which over-crowding and feed interruption emerge on the swine premises under movement restrictions during a classical swine fever outbreak. Animal 2017;11:493-499.
26. Yadav S, Weng HY. Estimating the scale of adverse animal welfare consequences of movement restriction and mitigation strategies in a classical swine fever outbreak. BMC Vet Res 2017;13:83.

27. North American Meat Institute. Significant events and progress involving the meat and poultry industry during the COVID-19 pandemic. Available at: www.meatinstitute.org/ht/a/ GetDocumentAction/i/179846. Accessed Dec 17, 2020.

\section{Appendix}

Protocol used by the farm described in the present report for depopulation of pigs because of packing plant closures caused by the COVID- 19 pandemic.

VSD+TH Protocol

VSD + TH cycle CANNOT be initiated or ended without approval from barn veterinarian.

Preparation

I) LogTag temperature and humidity recorders placed at each end of the barn.

2) Load pigs into the barn at approximately I,500 head (finishing pigs) or 3,500 to 4,000 head (nursery pigs).

3) Barn owner: validates that the room is clear of all people.

4) Barn owner: all clear is communicated via 2-way radios to all staff and in-barn veterinarian.

5) Barn veterinarian: initiates cycle START via 2-way radio communication.

6) All doors are shut.

7) Barn exhaust fans are sealed with fan covers.

Start Cycle

I) Barn veterinarian will be stationed in the control room.

2) Veterinarian will document all events throughout the cycle.

3) The veterinarian will begin a room timer for the barn.

4) Heaters turned on; no steam; beginning temperature recorded and recording of temperature throughout cycle.

5) At $54^{\circ} \mathrm{C}\left(130^{\circ} \mathrm{F}\right)$, time 0 , the veterinarian turns heaters off. a. Can restart heaters to maintain a temperature range of $49^{\circ} \mathrm{C}$ to $65^{\circ} \mathrm{C}\left(120^{\circ} \mathrm{F}\right.$ to $\left.150^{\circ} \mathrm{F}\right)$, as needed.

6) At $54^{\circ} \mathrm{C}$, time 0 , veterinarian requests steam generation.

7) Veterinarian monitors and records:

a. Room temperature.

b. Relative humidity.

c. Time to achieve $54^{\circ} \mathrm{C}$ in minutes from heat initiation.

d. The time when the room is silent (no pig movement, breathing, or vocalization).

8) Thirty minutes from time 0 , the veterinarian evaluates barn for pig movement or vocalization in 5 -minute intervals.

a. If movement or vocalization is noted, the cycle continues.

b. If no movement or vocalization is noted, the veterinarian will initiate END CYCLE (STOP) process.

End Cycle (STOP)

I) Veterinarian turns the heaters off, if operating, and communicates ALL STOP for steam generation.

2) Barn owner removes fan covers.

3) Veterinarian turns on fans from the control room.

4) All doors are opened.

5) The veterinarian visually evaluates the barn to ensure no animal movement while waiting for the barn's humidity to be ventilated.

6) Veterinarian, barn owner, and I additional person walk through the barn and evaluate each pig for any sign of consciousness.

a. If signs of consciousness (breathing, heartbeat, body movement, blinking, corneal reflex, or response to a painful stimulus) are seen, captive bolt euthanasia is applied immediately.

7) The veterinarian will declare the "all clear" after all pigs are verified as dead.

8) Carcass removal is permitted upon the veterinarian and all personnel exiting the building.

9) LogTag recorders are collected and identified to the barn on cycle record, records and LogTag devices are taken to the office, and the depopulation team will debrief at the end of each day. 